\title{
PROCESOS ACTUALES EN EL ESCARPE DE YESOS DE REMOLINOS
}

\author{
F. Pellicer* \\ M.T. Echeverría* \\ M.J. Ibáñez*
}

\begin{abstract}
RESUMEN
Entre los fenómenos funcionales que condicionan la evolución del escarpe de yesos de Remolinos (margen izquierda del Ebro) hemos seleccionado los movimientos en masa, destacando los ligados a desplazamientos con desplomes (slumps), favorecidos por la existencia de planos de fisuración paralelos al escarpe y por la eficacia de los procesos de disolución sobre los yesos.
\end{abstract}

\section{SUMMARY}

Among the functional phenomena that have an influence on the evolution of the gypsum bluff of Remolinos (left bank of the Ebro Riber), we have selected the mass movements, especially those linked to movements with slumps. These are encouraged by crack plans, parallel to the bluff and by the dissolution processes in the gypsums.

El escarpe de Remolinos se localiza en el sector central de la Depresión del Ebro, en la margen izquierda del río, en donde se extiende a lo largo de $36 \mathrm{kms}$. entre las desembocaduras del Gállego y del Arba. Con un desnivel que oscila entre los 40-60 m. en los extremos y los 186 en la Loma de Utebo, sobre el cauce actual del Ebro, este escarpe es el responsable de la disimetría topográfica y de depósitos aluviales en este sector del valle.

Los materiales en que se desarrolla pertenecen a un Mioceno medio, débilmente deformado por las últimas fases de la tectónica alpina, y coinci-

\footnotetext{
* Departamento de Geografía General. Universidad de Zaragoza.
} 
dentes con las denominadas por QUIRANTES (1969) Formación Alfocea y Formación Remolinos, constituida la primera por yesos, margas yesíferas y limos, y la segunda por limos, yesos, sales y algún paquete de caliza. Nuestras observaciones directas sobre el escarpe nos han permitido distinguir un nivel basal de margas aflorantes en el tramo oriental, al Este de la Granja de Santa Inés, al que se superpone un nivel de yesos generalizado en todo el sector estudiado y que presenta intercalaciones de sales en el área de Remolinos; hacia el techo aparece un nivel de margas rojizas que en el tramo de Remolinos posee yesos interestratificados, y que hacia el Oeste disminuye de potencia sirviendo de base a los niveles calcáreos (base de la Formación Castellar) de la Loma de Utebo y Mina Real.

Este componente litológico ha jugado un papel decisivo en la evolución cuaternaria del escarpe, condicionando por una parte fenómenos de disolución, particularmente importantes en los yesos, a la vez que la superposición de éstos a niveles impermeables margosos ha determinado la formación de planos de discontinuidad en cuanto al comportamiento mecánico de los materiales.

En origen se trata de un escarpe tectónico coincidente con líneas de fracturación Noroeste-Sureste, semejantes a las descritas por IBAÑEZ y MENSUA (1976) en el sector de Alfajarín, y relacionadas con fenómenos de neotectónica cuaternaria, con posibles rejuegos hasta la génesis de la $T$ II. No obstante, en el tramo de Remolinos la dinámica fluvial ha jugado un papel decisivo en el modelado del escarpe, lo que se traduce en la existencia de amplias concavidades relacionadas con antiguos meandros, que desdibujan la linealidad del escarpe, y que no se constatan en el caso de Alfajarín.

\section{Vertientes con movimientos en masa}

Entre los procesos recientes y funcionales hemos seleccionado los movimientos en masa, que si no son los únicos, son los más representativos en el modelado del escarpe. Así pues, hemos relegado conscientemente procesos tales como incisiones fluviales, movimientos individuales de partículas (saltación y reptación), y otros de origen biológico, y que serán abordados en un trabajo más amplio en curso de realización.

\section{Vertientes con desplome y desplazamiento (slump y earthflow)}

Los desplomes funcionales de este tipo afectan bien al escarpe original (Ruinas de Santa Inés, Ermita del Castellar), en cuyo caso poseen una planta en forma de huso y unas dimensiones de 200 por $40 \mathrm{~m}$. aproximadamente, bien a masas rocosas previamente movilizadas por mecanismos semejantes a los que describimos pero de mayor escala (hasta 100 por 250 m.) (Mejana Redonda). 
Este tipo de vertiente se localiza fundamentalmente, de Este a Oeste, en el Galacho de Juslibol, en el sector de Mejana Redonda (al Oeste de Alfocea), en el sector de la Acequia del Terraplén (Norte de Sobradiel), en la Ermita del Castellar (Norte de Torres de Berrellén) y en las Ruinas de Santa Inés (Norte de Alagón), coincidiendo su ubicación con las partes cóncavas de los meandros tangentes al escarpe.

Se trata de vertientes de perfil complejo, con secciones convexas, más o menos estabilizadas y superpuestas en la vertiente. A pesar de esta complejidad cabe distinguir en general una parte inferior convexa, con forma lingüoide en planta, con bloques dispuestos caóticamente y grietas radiales surcando la acumulación, y una parte superior en la que se han deslizado paquetes a partir de un plano de discontinuidad, y que conservan su estructura tras el deslizamiento rotacional a que han sido sometidos, tal como se deduce del buzamiento a contrapendiente que presentan los estratos integrantes del paquete.

Aunque estas características son comunes a todos los puntos señalados, la morfología, modelado y escala del fenómeno difiere, estando ello en relación con la funcionalidad o no del fenómeno.

En el caso de vertientes funcionales del desplome y consiguiente acumulación deriva de una compleja interferencia de hechos, entre los que cabe destacar los siguientes:

a) Presencia de líneas de diaclasación asociadas a la fractura mayor que ha determinado el escarpe original. Estas diaclasas constituyen planos de debilidad dentro de la masa rocosa, resultando favorables al desplazamiento mecánico de paquetes y a la acción hídrica, y condicionando procesos de meteorización y disolución.

b) Fenómenos de disolución que se traducen en:

- Dolinas-pozo, localizadas en los puntos de cruce o interferencia de diaclasas y en las que se puede diferenciar un embudo en la parte superior, cuyas dimensiones oscilan alrededor de los 4-6 m. de diámetro y 1,5 m. de profundidad, y un cuello en la base de unos $13 \mathrm{~m}$. de longitud y $2 \mathrm{~m}$. de anchura. El fondo de estas dolinas queda obturado por un terrón que tapona cavidades internas.

Este tipo de dolinas se alinea en cordones paralelos al escarpe y a diferentes niveles topográficos. La coalescencia de dolinas en algunos puntos, a partir de dicha alineación, es lo que favorece precisamente el despeque de paneles en la vertiente. Una vez individualizado el paquete rocoso, el plano de despegue presenta un modelado en "medias cañas", resultado de la morfología de las dolinas que han jugado como motor del desplome.

- Pequeñas cubetas de 2 a $3 \mathrm{~m}$. de diámetro, generadas por procesos asociados de disolución, subfusión y arrastre de partículas, y localizadas en 
las áreas en las que abundan limos y formaciones edáficas. En muchos casos estas cubetas constituyen el estadio inicial del tipo anterior.

- Apertura de grietas de diaclasación que por disolución tienden a ensancharse progresivamente.

Todos estos fenómenos son funcionales en la actualidad en relación con una infiltración de las aguas y un drenaje interno de las formaciones. El caso más expresivo, aunque no está directamente ligado al tipo de vertientes que ahora describimos, lo hemos encontrado en la resurgencia de Ojo Salado, al Oste de Remolinos, relacionada con el área de dolinas localizada en la parte superior del escarpe, de mayores dimensiones que las descritas $(60 \mathrm{~m}$. de diámetro y $20 \mathrm{~m}$. de profundidad), y que en este momento no condicionan la evolución del escarpe.

c) Acción fluvial, que funciona como agente dinamizador de procesos, y que es efectiva tanto por impacto de la corriente fluvial sobre el escarpe, socavando la base del mismo y favoreciendo así la movilización de las masas rocosas deslizadas en la vertiente, como por humectación de la roca. No obstante, los efectos son distintos en función de la litología.

Así, en el sector de las Ruinas de Santa Inés la base de material margoso va perdiendo coherencia a partir de la humectación de origen vertical, a través del agua que filtran las grietas, y horizontal, a partir del contacto del mismo río. Como resultado la masa rocosa adquiere un estado plástico en el paquete inferior que, presionado por el paquete superior, se deforma formándose pequeñas "coladas de tierra" ("earthflow", VARNES, 1978). Pero en los tramos del Galacho de Juslibol y Mejana Redonda, al Oeste de Alfocea, donde la base está formada por yesos, la presión de los paquetes superiores y la acción del río socavando la base del escarpe originan el derrumbamiento de parte de éste, formándose un talud caótico de acumulación de bloques que morfológicamente guarda cierta similitud con las "coladas de tierra" del sector de las Ruinas de Santa Inés. En este caso la humectación se limita a favorecer el desmembramiento de los niveles de base en bloques.

Dentro del tipo de vertientes con desplomes, éstas pueden presentar una gran complejidad y un aspecto marcadamente caótico; es el caso del tramo al Oeste de Alfocea, en donde la vertiente se ha descompuesto en múltiples paneles funcionales, superpuestos unos a otros, aunque de tamaño mucho más reducido que en los de las vertientes anteriormente citadas.

\section{Vertientes con "slump"}

En las proximidades de Juslibol, en la Granja de la Pola y en la Partida de Cueva del Topo, como puntos más significativos, se constata un tipo de vertiente de evolución más elemental que las antes descritas, y en las que 
aparecen pequeños paquetes individualizados y despegados por movimiento rotacional.

En nuestra opinión, y dadas las reducidas dimensiones de los paquetes no se ejerce una presión importante sobre la base, por lo que no se constatan las coladas de tierra que aparecían en otros tramos de vertiente.

La litología en que aparece este tipo de vertiente es diversa, de donde se deduce que su génesis no queda condicionada por este factor.

\section{Vertientes con bloques}

Agrupamos dentro de esta denominación vertientes con características distintas, pero que tienen en común la presencia de acumulaciones de bloques ligadas a desprendimientos masivos.

a) Vertientes con avalanchas de bloques; por ejemplo, la vertiente localizada al norte de Torres de Berrellén, en la Partida del Cerro del Pillo, en donde se constata un desprendimiento cuyas dimensiones alcanzan los 350 $\mathrm{m}$. de largo por $150 \mathrm{~m}$. de ancho y $20 \mathrm{~m}$. de altura. Su zona de arranque se identifica con una cicatriz suavemente cóncava en planta, que se corresponde con una discontinuidad mecánica de plano vertical, a partir de la cual se ha movilizado la masa de la vertiente, caracterizada en la base por una vacumulación de bloques, aun cuando en la parte más oriental se han desprendido paquetes de comportamiento más homogéneo, lo que impone un aspecto diferente a la morfología del conjunto. Parece tratarse de un tramo de vertiente de génesis brusca y reciente, dado el aspecto fresco de la cicatriz, la cual se halla encajada en otra de dimensiones mayores que dejan colgadas vales, y dado la invasión del lecho fluvial por la propia masa derrubiada que deforma la curvatura natural de la orilla cóncava del río.

b) Vertientes con acumulación de bloques formando cono. Se trata de derrumbes diferenciados de los anteriores por la forma en planta de la acumulación así como por las dimensiones de la misma, puesto que en este caso son más reducidas.

Este tipo de fenómeno, muy puntual, está ligado al desprendimiento de bloques a partir de una cornisa, constatándose una cierta ordenación por tamaños del material. No obstante, por las propias características del roquedo no han llegado a generarse formas típicas, hecho que también ha quedado impedido en algún caso por el abrupto perfil de las vertientes, que al Oeste de Alfocea alcanza una pendiente de $45-50^{\circ} \mathrm{C}$.

Junto a estos tipos de vertientes ligados a desprendimientos masivos, existen también vertientes con bloques de simple gravedad. Constituyen éstas un tipo más elemental y muy generalizado que se desarrolla tanto sobre el propio escarpe original como sobre otros tipos de vertientes ya descritas. Sus dimensiones, en muchos casos de escala métrica indican que se trata de 
retoques menores sobre morfologías preexistentes. Son fácilmente identificables por la escasa meteorización que presenta tanto el área de arranque como los propios bloques desprendidos, los cuales normalmente se desmembran en fragmentos de menor tamaño al chocar con la base.

\section{Conclusiones}

La originalidad de los movimientos en masa que acabamos de describir radica en la interacción de una serie de hechos:

- La potente formación de yesos en los que se ha generado el escarpe y a la que quedan ligados básicamente estos desprendimientos. Precisamente el carácter mașivo de los yesos imprime una particular morfología a la vertiente, por lo que no siempre resulta fácil aplicar un modelo teórico.

- El carácter soluble de estos materiales yesíferos, que conlleva el desarrollo de fenómenos Kársticos, con génesis de dolinas y ampliación de diaclasas, lo que favorece la circulación hídrica y la humectación de la base del escarpe, todo lo cual acelera los desplomes.

- La proximidad del curso fluvial al escarpe ejerciendo un doble papel; por una parte el contacto con el agua modifica el estado hidrométrico de las formaciones litológicas de base y por otra el arrastre de material por el río y el impacto de la corriente sobre el pie del escarpe produce variaciones en las condiciones de estabilidad.

La funcionalidad de estas vertientes en la actualidad es evidente, tal como lo demuestran una serie de observaciones: la invasión puntual del lecho del río imprimiendo curvaturas forzadas a la orilla convexa del meandro; el aspecto fresco que presenta en muchos casos la cicatriz de arranque, todavía no retocada por incisiones de barrancos ni afectada por fenómenos de meteorización superficial; el hecho de que yacimientos arqueológicos ibéricos y medievales estén parcialmente destruidos, como en el caso de Salduva y del castillo de Miranda en Juslibol; el que dos raíces de un mismo arbusto se encuentren "a caballo" de una fisura afectando a un bloque en vías de desprendimiento, como se ha podido constatar en el sector de las Ruinas de Santa Inés; y por último, la funcionalidad de los fenómenos Kársticos queda patente en la señalada resurgencia de Ojo Salado. 


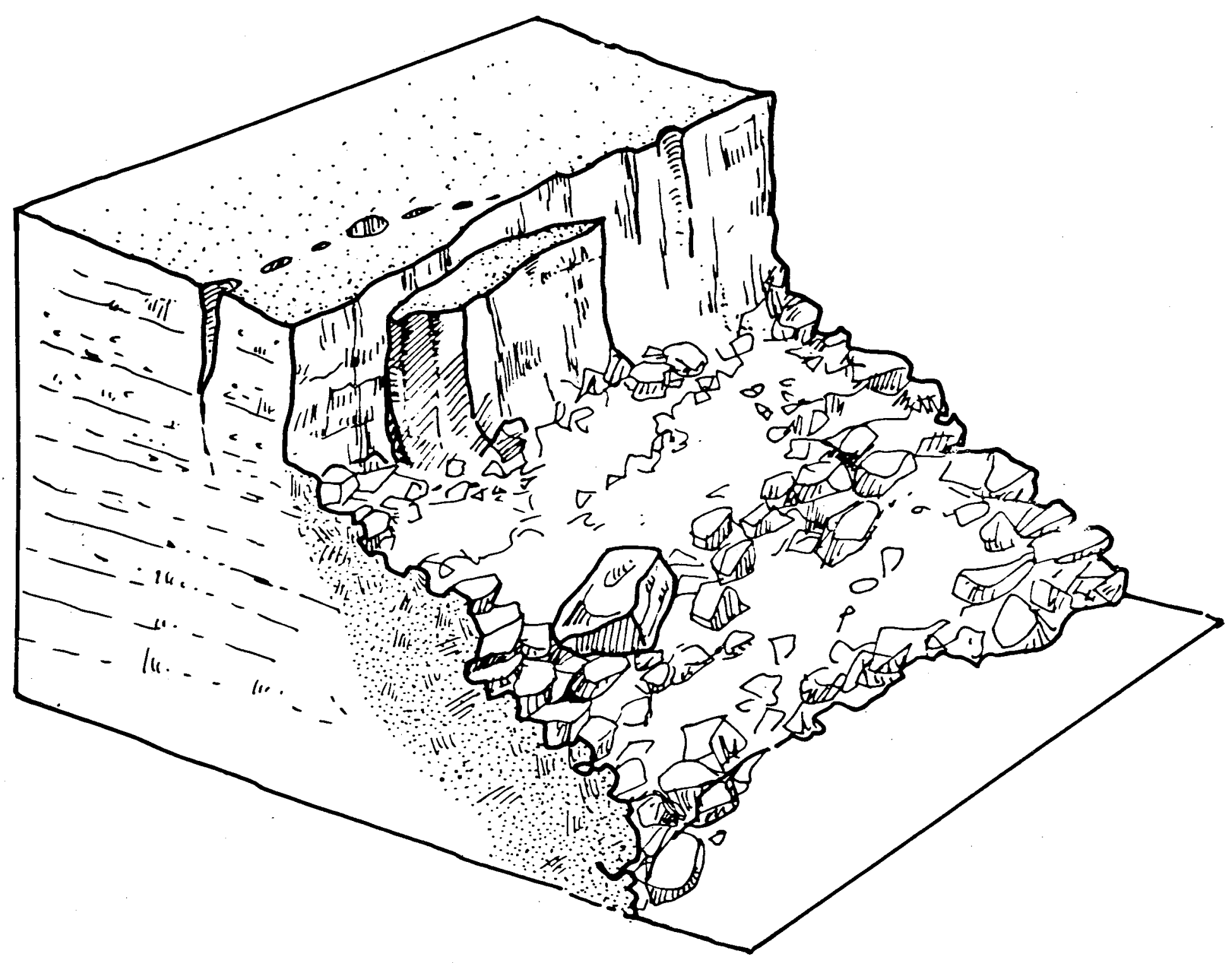

Vertiente con desplome y desplazamiento. En el escarpe se aprecian las "medias cañas". Inicio de paneles a partir de alineación de pequeñas dolinas. 


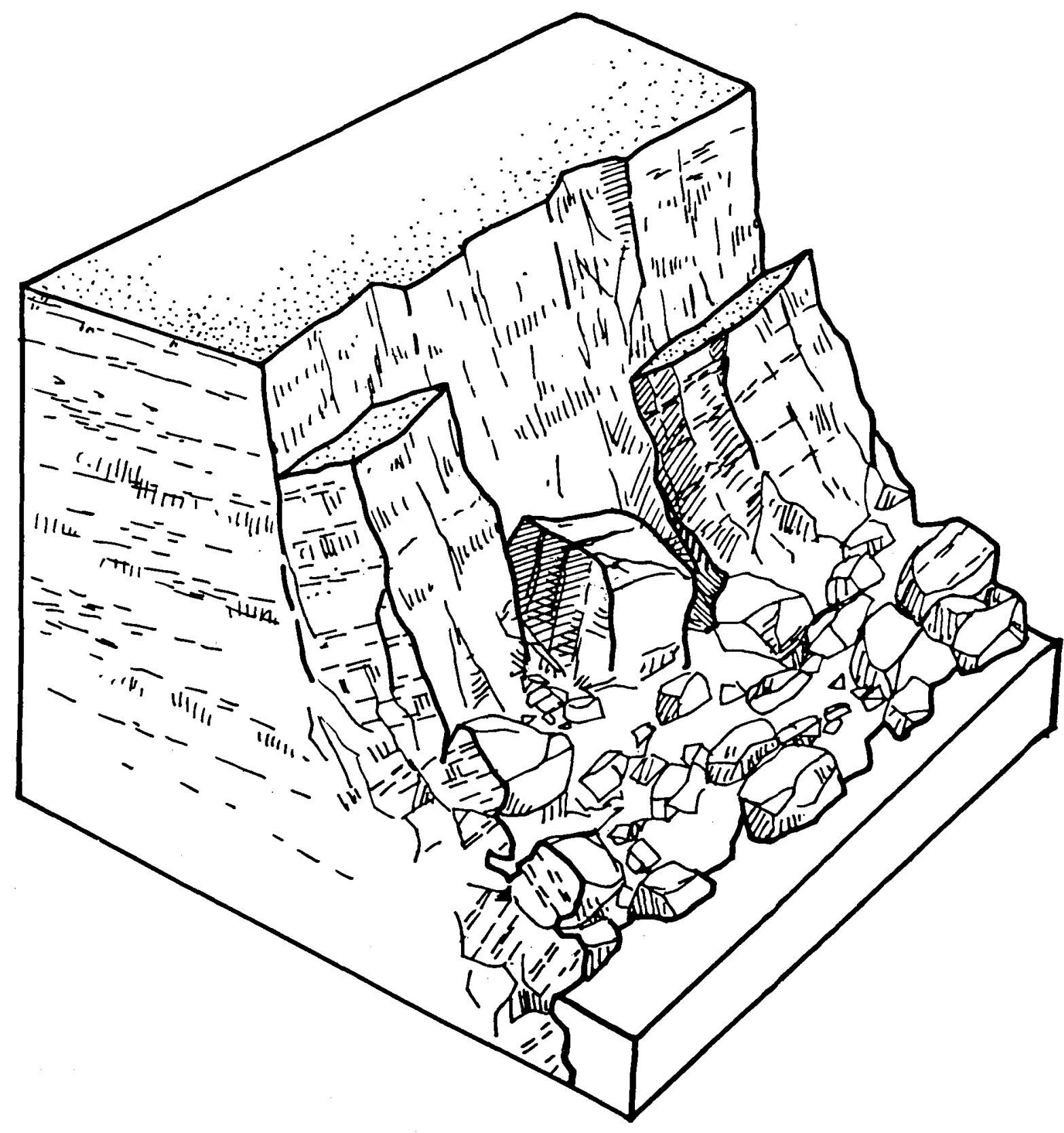

Vertiente con desplome de paneles y desplazamiento. 
PROCESOS ACTUALES EN EL ESCARPE DE YESOS DE REMOLINOS

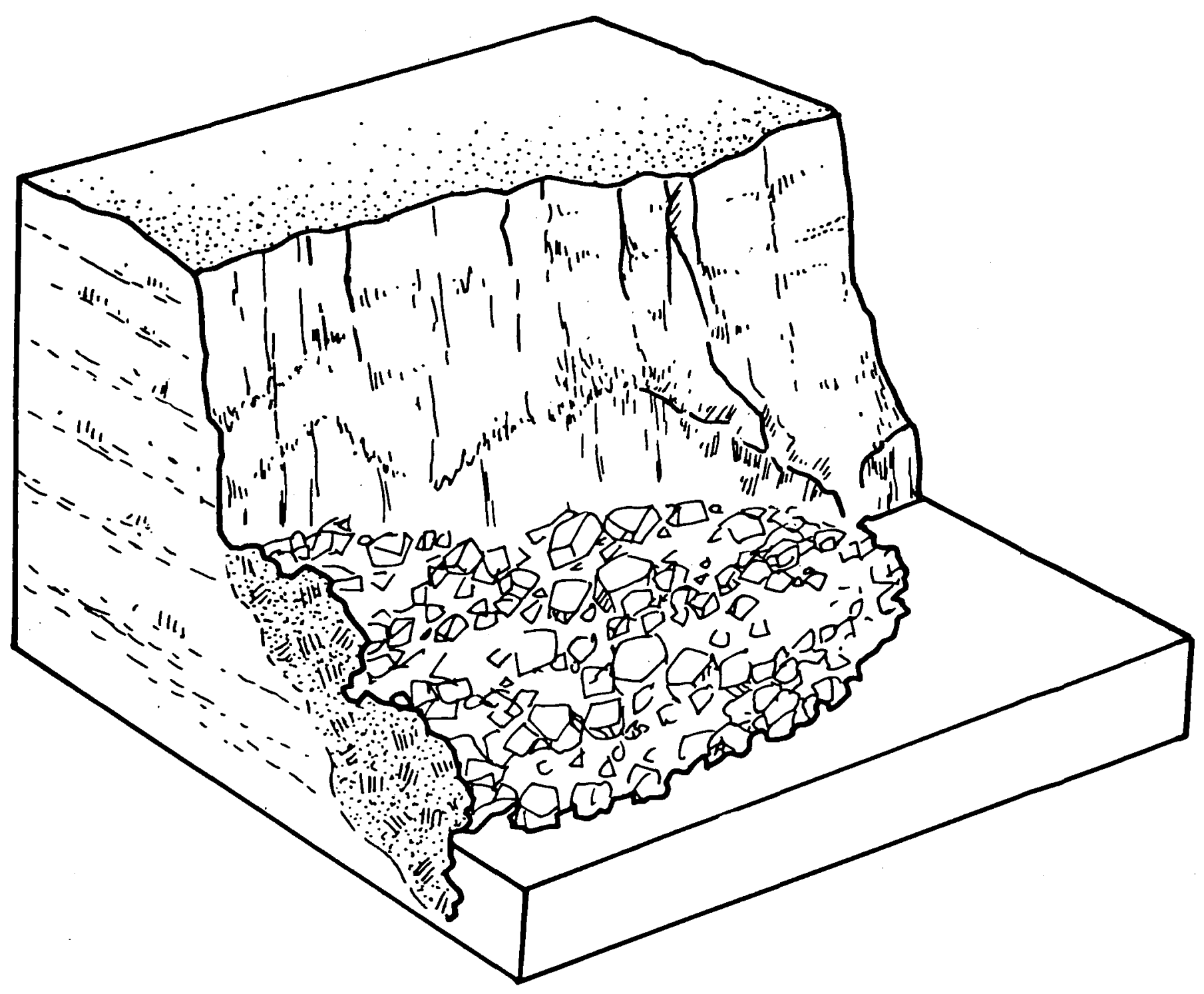

Vertiente con acumulación de bloques. Sector norte de Torres de Berrellén. 


\section{BIBLIOGRAFIA}

YOUNG, A. (1975). Slopes. Longman Group Limited. Geomorphology text; 3. New York.

IBAÑEZ, M.J. y MENSUA, S. (1976). Contribución al estudio de vertientes en condiciones semiáridas: Tipos de vertientes sobre yesos en el valle del Ebro. Boletín de la Real Sociedad Geográfica. Tomo CXII. Parte II. Madrid.

QUIRANTES, F. (1978). Estudio sedimentológico y estratigráfico del Terciario continental de los Monegros. Institución Fernando el Católico. C.S.I.C. Zaragoza.

TRANSPORTATION RESEARCH BOARDS. Commission on Sociotechnical Systems National Research Council, 1978: Lanslides, Analysis and Control. National Academy of Sciences. Washington.

VARNES, D.J. (1978). Slope Movement and Types and Processes. In SCHUSTER, R.L. y KRIZER, R.J. Edit.: Landslides. Analysis and Control. National Academy of Sciences. Washington. 